\title{
ON CERTAIN SUBCLASS OF P-VALENT MEROMORPHICALLY STARLIKE FUNCTIONS WITH ALTERNATING COEFFICIENTS
}

\author{
H. E. DARWISH, A. Y. LASHIN AND B. H. RIZQAN
}

\begin{abstract}
A certain subclass $B_{m}(p, \alpha, \lambda, \ell, A, B)$ consisting of meromorphic p-valent functions with alternating coefficient in $U^{*}=\{z: z \in C: 0<|z|<1\}$ is introduced. In this paper we obtain coefficient inequalities, distortion theorem, closure theorems and class preserving integral operators for functions in the class $B_{m}(p, \alpha, \lambda, \ell, A, B)$ are obtained.
\end{abstract}

\section{Introduction}

Let $\Sigma(p)$ denote the class of functions of the form:

$$
f(z)=\frac{a_{-p}}{z^{p}}+\sum_{k=1}^{\infty} a_{k} z^{k} \quad\left(a_{-p} \neq 0 ; p \in \mathbb{N}:=\{1,3,5, \ldots\}\right),
$$

which are regular in the punctured disc $U^{*}=\{z: z \in C: 0<|z|<1\}=U \backslash\{0\}$, see [11].

Definition 1. Let $f, g$ be analytic in $U$. Then $g$ is said to be subordinate to $f$, written $g \prec f$, if there exists a Schwarz function $w(z)$, which is analytic in $U$, with $w(0)=0$ and $|w(z)|<$ $1(z \in U)$ such that $g(z)=f(w(z))(z \in U)$. Hence $g(z) \prec f(z)(z \in U)$, then $g(0)=f(0)$ and $g(U) \subset f(U)$.In particular, if the function $f(z)$ is univalent in $U$, we have the following (e.g. [7]; [8]):

$g(z) \prec f(z)(z \in U)$ if and only if $g(0)=f(0)$ and $g(U) \subset f(U)$.

Definition 2. For functions $f(z) \in \sum(p)$ given by (1.1) and $g(z) \in \sum(p)$ defined by

$$
g(z)=\frac{b_{-p}}{z^{p}}+\sum_{k=1}^{\infty} b_{k} z^{k} \quad\left(b_{k} \geq 0, p \in \mathbb{N}\right),
$$

we define the convolution (or Hadamard product) of $f(z)$ and $g(z)$ by

$$
(f * g)(z)=\frac{a_{-p} b_{-p}}{z^{p}}+\sum_{k=1}^{\infty} a_{k} b_{k} z^{k} \quad(p \in \mathbb{N}, z \in U) .
$$

Received April 23, 2013, accepted November 15, 2013.

2010 Mathematics Subject Classification. Primary 30C45.

Key words and phrases. Regular, meromorphic functions, starlike functions, distortion theorem, closure theorem, integral operators. 
Now, using the integral operator $L_{p}^{m}(\lambda, \ell) \quad\left(\ell>0 ; \lambda \geq 0 ; p \in \mathbb{N} ; m \in N_{0}=N \cup\{0\}=\right.$ $\left.\{0,1,2, \ldots\}, z \in U^{*}\right)$ introduced by El-Ashwah [4], the for function $f(z) \in \sum(p)$ given by (1.1) as follows:

$$
L_{p}^{m}(\lambda, \ell) f(z)=\frac{a_{-p}}{z^{p}}+\sum_{k=1}^{\infty}\left[\frac{\ell}{\ell+\lambda(k+p)}\right]^{m} a_{k} z^{k} .
$$

It is easily verified from (1.4) that

$$
\left.\lambda z\left(L_{p}^{m+1}(\lambda, \ell)\right) f(z)\right)^{\prime}=\ell L_{p}^{m}(\lambda, \ell) f(z)-(\ell+p \lambda) L_{p}^{m+1}(\lambda, \ell) f(z)(\lambda>0),
$$

we note that:

(i) $L_{p}^{\alpha}(1,1) f(z)=p_{p}^{\alpha} f(z)$ (see Aqlan et al. [3]);

(ii) $L_{1}^{\alpha}(1, \beta) f(z)=p_{\beta}^{\alpha} f(z)\left(a_{-p}=1\right)$ (see Lashin [6]);

(iii) $L(1, \gamma) f(z)=J(f(z))(p=1)$ (see Sh. Najafzadeh [9]);

(iv) $L_{p}^{m}(1, \alpha) f(z)=J_{p, \alpha}^{m} f(z)$ (see El -Ashwah et. at [5]).

Let $B_{m}(p, \alpha, \lambda, \ell, A, B)$ denote the class of functions $f(z)$ in $\sum(p)$ that satisfy the condition :

$$
\frac{\ell L_{p}^{m}(\lambda, \ell) f(z)}{\lambda L_{p}^{m+1}(\lambda, \ell) f(z)}-\left(\frac{\lambda p+\ell}{\lambda}\right)<-\frac{p+[p B+(A-B)(p-\alpha)] z}{1+B z}, \quad z \in U^{*}
$$

where $<$ denotes subordination, $0 \leq \alpha<p,-1 \leq A<B \leq 1,0<B \leq 1, \lambda, \ell>0, p \in \mathbb{N}$ and $m \in \mathbb{N}_{0}$.

By definition of subordination, the condition (1.6) is equivalent to

$$
\frac{\ell L_{p}^{m}(\lambda, \ell) f(z)}{\lambda L_{p}^{m+1}(\lambda, \ell) f(z)}-\left(\frac{\lambda p+\ell}{\lambda}\right)=\frac{p+[p B+(A-B)(p-\alpha)] w(z)}{1+B w(z)},
$$

where $w(z) \in H=\{w$ regular, $w(0)=0$ and $|w(z)|<1, z \in U\}$. It is easy to see that the condition (1.7) is equivalent to

$$
\left|\frac{\frac{\ell L_{p}^{m}(\lambda, \ell) f(z)}{\lambda L_{p}^{m+1}(\lambda, \ell) f(z)}-\frac{\ell}{\lambda}}{B\left[\frac{\ell L_{p}^{m}(\lambda, \ell) f(z)}{\lambda L_{p}^{m+1}(\lambda, \ell) f(z)}-\left(\frac{\lambda p+\ell}{\lambda}\right)\right]+[p B+(A-B)(p-\alpha)]}\right|<1 \quad\left(z \in U^{*}\right)
$$

We note that:

(i) when $A=-1, B=1$, we have $f(z) \in B_{m}(p, \alpha, \lambda, \ell)$ if

$$
\operatorname{Re}\left\{\frac{\ell L_{p}^{m}(\lambda, \ell) f(z)}{\lambda L_{p}^{m+1}(\lambda, \ell) f(z)}-\left(\frac{\lambda p+\ell}{\lambda}\right)\right\}<-\alpha
$$


(ii) when $\alpha=0$

$$
\frac{\ell L_{p}^{m}(\lambda, \ell) f(z)}{\lambda L_{p}^{m+1}(\lambda, \ell) f(z)}-\left(\frac{\lambda p+\ell}{\lambda}\right) \prec-\frac{1+A z}{1+B z} .
$$

Let $\sum_{a}(p)$ be the subclass of $\sum(p)$ consists of functions of the form:

$$
f(z)=\frac{a_{-p}}{z^{p}}+\sum_{k=1}^{\infty}(-1)^{k-1} a_{k} z^{k} \quad\left(a_{-p} \neq 0 ; a_{k} \geq 0 ; p \in \mathbb{N}\right),
$$

that are regular and p-valent in $U^{*}$.

Let us write

$$
\sum_{a}^{*}(p, m, \alpha, \lambda, \ell, A, B)=B_{m}(p, \alpha, \lambda, \ell, A, B) \cap \sum_{a}(p) .
$$

In this paper coefficient inequalities, distortion theorem and closure theorems for the class $B_{m}^{*}(p, \alpha, \lambda, \ell, A, B)$ are obtained.

Finally, the class preserving integral operators of the form

$$
F(z)=(c-p+1) z^{-c-1} \int_{0}^{z} t^{c} f(t) d t \quad\left(c>p-1 ; p \in N_{0}\right),
$$

is considered. Techinques used are similar to those of Silvermen [10] and Uralegaddi and Ganigi [11], Aouf and Darwish [1], Aouf et al. [2].

\section{Coefficient inequalities}

Theorem 1. Let $f(z)=\frac{a_{-p}}{z^{p}}+\sum_{k=1}^{\infty} a_{k} z^{k}$ be regular and p-valent in $U^{*}$. If

$$
\begin{aligned}
& \sum_{k=1}^{\infty}\left[\frac{\ell}{\ell+\lambda(k+p)}\right]^{m+1}[(k+p)(1+B)+(A-B)(p-\alpha)]\left|a_{k}\right| \\
& \quad \leq(B-A)(p-\alpha)\left|a_{-p}\right|,
\end{aligned}
$$

then $f(z) \in B_{m}(p, \alpha, \lambda, \ell, A, B)$.

Proof. Suppose (2.1) holds for all admissible values of $p, m, \alpha, \lambda, \ell, A$ and $B$. It suffices show that

$$
\left|\frac{\frac{L_{p}^{m}(\lambda, \ell) f(z)}{L_{p}^{m+1}(\lambda, \ell) f(z)}-1}{B\left[\frac{L_{p}^{m}(\lambda, \ell) f(z)}{L_{p}^{m+1}(\lambda, \ell) f(z)}-\left(\frac{\lambda}{\ell} p+1\right)\right]+\lambda / \ell[p B+(A-B)(p-\alpha)]}\right|<1 \text { for }|z|<1
$$

we have

$$
\left|\frac{\frac{L_{p}^{m}(\lambda, \ell) f(z)}{L_{p}^{m+1}(\lambda, \ell) f(z)}-1}{B\left[\frac{L_{p}^{m}(\lambda, \ell) f(z)}{L_{p}^{m+1}(\lambda, \ell) f(z)}-\left(\frac{\lambda}{\ell} p+1\right)\right]+\lambda / \ell[p B+(A-B)(p-\alpha)]}\right|
$$




$$
\begin{aligned}
& =\left|\frac{\sum_{k=1}^{\infty}\left[\frac{\ell}{\ell+\lambda(k+p)}\right]^{m+1}(k+p) a_{k} z^{p+k}}{(A-B)(p-\alpha) a_{-p}+\sum_{k=1}^{\infty}\left[\frac{\ell}{\ell+\lambda(k+p)}\right]^{m+1}[B(k+p)+(A-B)(p-a)] a_{k} z^{k+p}}\right| \\
& \leq \frac{\sum_{k=1}^{\infty}\left[\frac{\ell}{\ell+\lambda(k+p)}\right]^{m+1}(k+p)\left|a_{k}\right|}{(B-A)(p-\alpha)\left|a_{-p}\right|-\sum_{k=1}^{\infty}\left[\frac{\ell}{\ell+\lambda(k+p)}\right]^{m+1}[B(k+p)+(A-B)(p-a)]\left|a_{k}\right|} .
\end{aligned}
$$

The last expression is bounded above by (1.1) if

$$
\begin{aligned}
\sum_{k=1}^{\infty}\left[\frac{\ell}{\ell+\lambda(k+p)}\right]^{m+1}(k+p)\left|a_{k}\right| \leq(B-A)(p-a)\left|a_{-p}\right| \\
\quad-\sum_{k=1}^{\infty}\left[\frac{\ell}{\ell+\lambda(k+p)}\right]^{m+1}[B(k+p)+(A-B)(p-\alpha)]\left|a_{k}\right|
\end{aligned}
$$

which is equivalent to

$$
\sum_{k=1}^{\infty}\left[\frac{\ell}{\ell+\lambda(k+p)}\right]^{m+1}[(k+p)(1+B)+(A-B)(p-\alpha)]\left|a_{k}\right| \leq(B-A)(p-\alpha)\left|a_{-p}\right| .
$$

The completes the proof of Theorem 1 .

Theorem 2. Let $f(z)=\frac{a_{-p}}{z^{p}}+\sum_{k=1}^{\infty}(-1)^{k-1} a_{k} z^{k} \quad\left(a_{-p} \neq 0 ; a_{k} \geq 0, p \in \mathbb{N}\right)$ be regular and $p$ valent in $U^{*}$. Then $f(z) \in \Sigma_{a}^{*}(p, m, \alpha, \lambda, \ell, A, B)$ if and only if

$$
\begin{aligned}
& \sum_{k=1}^{\infty}\left[\frac{\ell}{\ell+\lambda(k+p)}\right]^{m+1}[(k+p)(1+B)+(A-B)(p-\alpha)] a_{k} \\
& \quad \leq(B-A)(p-\alpha)\left|a_{-p}\right| .
\end{aligned}
$$

Proof. In view of Theorem 1, it is sufficient to shwo the "only if " part. Let us suppose that

$$
f(z)=\frac{a_{-p}}{z^{p}}+\sum_{k=1}^{\infty}(-1)^{k-1} a_{k} z^{k}\left(a_{-p} \neq 0 ; a_{k} \geq 0, p \in \mathbb{N}\right),
$$

is in $\sum_{a}^{*}(p, m, \alpha, \lambda, \ell, A, B)$. Then

$$
\begin{array}{|c}
\left|\frac{\frac{L_{p}^{m}(\lambda, \ell) f(z)}{L_{p}^{m+1}(\lambda, \ell) f(z)}-1}{B\left[\frac{L_{p}^{m+1}(\lambda, \ell) f(z)}{L_{p}^{m+1}(\lambda, \ell) f(z)}-\left(\frac{\lambda}{\ell} p+1\right)\right]+\frac{\lambda}{\ell}[p B+(A-B)(p-\alpha)]}\right| \\
\quad=\left|\frac{\sum_{k=1}^{\infty}(-1)^{k-1}\left[\frac{\ell}{\ell+\lambda(k+p)}\right]^{m+1}(k+p) a_{k} z^{k+p}}{(B-A)(p-\alpha) a_{-p}-\sum_{k=1}^{\infty}(-1)^{k-1}\left[\frac{\ell}{\ell+\lambda(k+p)}\right]^{m+1}[B(k+p)+(A-B)(p-\alpha)] a_{k} z^{k+p}}\right| \leq 1
\end{array}
$$


for all $z \in U^{*}$. Using the fact that $\operatorname{Re}(z) \leq|z|$ for all $z$, it follows that

$\operatorname{Re}\left\{\frac{\sum_{k=1}^{\infty}(-1)^{k-1}\left[\frac{\ell}{\ell+\lambda(k+p)}\right]^{m+1}(k+p) a_{k} z^{k+p}}{(B-A)(p-\alpha) a_{-p}-\sum_{k=1}^{\infty}(-1)^{k-1}\left[\frac{\ell}{\ell+\lambda(k+p)}\right]^{m+1}[B(k+p)+(A-B)(p-\alpha)] a_{k} z^{k+p}}\right\}$ $\leq 1\left(z \in U^{*}\right)$. (2.3)

Now choose values of $z$ on the real axis so that $\frac{L_{p}^{m}(\lambda, \ell) f(z)}{L_{p}^{m+1}(\lambda, \ell) f(z)}$ is real. Upon clearing the denominator in (2.3) and letting $z \rightarrow(-1)^{+}$through real values, we obtain

$$
\begin{aligned}
& \sum_{k=1}^{\infty}\left[\frac{\ell}{\ell+\lambda(k+p)}\right]^{m+1}(k+p) a_{k} \leq(B-A)(p-\alpha)\left|a_{-p}\right| \\
& -\sum_{k=1}^{\infty}\left[\frac{\ell}{\ell+\lambda(k+p)}\right]^{m+1}[B(k+p)+(A-B)(p-\alpha)] a_{k} .
\end{aligned}
$$

or

$$
\sum_{k=1}^{\infty}\left[\frac{\ell}{\ell+\lambda(k+p)}\right]^{m+1}[(k+p)(1+B)+(A-B)(p-\alpha)] a_{k} \leq(B-A)(p-\alpha)\left|a_{-p}\right| .
$$

Hence the result follows.

Corollary 1. If $f(z)=\frac{a_{-p}}{z^{p}}+\sum_{k=1}^{\infty}(-1)^{k-1} a_{k} z^{k} \quad\left(a_{-p} \neq 0 ; a_{k} \geq 0 ; p \in \mathbb{N}\right)$ is in the class $\sum_{a}^{*}(p, \alpha, \lambda, \ell, A, B)$, then

$$
a_{k} \leq \frac{(B-A)(p-\alpha)\left|a_{-p}\right|}{\left[\frac{\ell}{\ell+\lambda(k+p)}\right]^{m+1}[(k+p)(1+B)+(A-B)(p-\alpha)]}(k \in N) .
$$

Equality holds for the functions of form

$$
f_{k}(z)=\frac{a_{-p}}{z^{p}}+(-1)^{k-1} \frac{(B-A)(p-\alpha) a_{-p}}{\left[\frac{\ell}{\ell+\lambda(k+p)}\right]^{m+1}[(k+p)(1+B)+(A-B)(p-\alpha)]} z^{k},
$$

where $(k \in N$ and $p \in \mathbb{N})$.

\section{Distortion theorems}

Theorem 3. Let $f(z)=\frac{a_{-p}}{z^{p}}+\sum_{k=1}^{\infty}(-1)^{k-1} a_{k} z^{k}\left(a_{-p} \neq 0 ; a_{k} \geq 0 ; p \in \mathbb{N}\right)$ is in the class $\sum_{a}^{*}(p, m, \alpha, \lambda, \ell, A, B)$, then for $0<|z|=r<1$,

$$
\frac{\left|a_{-p}\right|}{r^{p}}-\frac{(B-A)(p-\alpha)\left|a_{-p}\right|}{\left[\frac{\ell}{\ell+\lambda(1+p)}\right]^{m+1}[(1+p)(1+B)+(A-B)(p-\alpha)]} r \leq|f(z)|
$$




$$
\leq \frac{\left|a_{-p}\right|}{r^{p}}+\frac{(B-A)(p-\alpha)\left|a_{-p}\right|}{\left[\frac{\ell}{\ell+\lambda(1+p)}\right]^{m+1}[(1+p)(1+B)+(A-B)(p-\alpha)]} r
$$

with equality for the function

$$
f_{1}(z)=\frac{a_{-p}}{z^{p}}+\frac{(B-A)(p-\alpha)\left|a_{-p}\right|}{\left[\frac{\ell}{\ell+\lambda(1+p)}\right]^{m+1}[(1+p)(1+B)+(A-B)(p-\alpha)]} z
$$

at $z=r, i r$.

Proof. Suppose that $f(z)$ in the class $\sum_{a}^{*}(p, m, \alpha, \lambda, \ell, A, B)$. In view of Theorem 2 , we have

$$
\begin{aligned}
& {\left[\frac{\ell}{\ell+\lambda(1+p)}\right]^{m+1}[(1+p)(1+B)+(A-B)(p-\alpha)] \sum_{k=1}^{\infty} a_{k}} \\
& \quad \leq \sum_{k=1}^{\infty}\left[\frac{\ell}{\ell+\lambda(k+p)}\right]^{m+1}[(1+p)(1+B)+(A-B)(p-\alpha)] a_{k} \\
& \quad \leq(B-A)(p-\alpha)\left|a_{-p}\right|,
\end{aligned}
$$

which evidently yields

$$
\sum_{k=1}^{\infty} a_{k} \leq \frac{(B-A)(p-\alpha)\left|a_{-p}\right|}{\left[\frac{\ell}{\ell+\lambda(1+p)}\right]^{m+1}[(1+p)(1+B)+(A-B)(p-\alpha)]} .
$$

Consequently, we obtain

$$
\begin{aligned}
|f(z)| & \leq \frac{\left|a_{-p}\right|}{r^{p}}+\sum_{k=1}^{\infty} a_{k} r^{k} \leq \frac{\left|a_{-p}\right|}{r^{p}}+r \sum_{k=1}^{\infty} a_{k} \\
& \leq \frac{\left|a_{-p}\right|}{r^{p}}+\frac{(B-A)(p-\alpha)\left|a_{-p}\right|}{\left[\frac{\ell}{\ell+\lambda(1+p)}\right]^{m+1}[(1+p)(1+B)+(A-B)(p-\alpha)]} r
\end{aligned}
$$

by (3.3). This gives the right hand inequality of (3.1). Also

$$
\begin{aligned}
|f(z)| & \geq \frac{\left|a_{-p}\right|}{r^{p}}-\sum_{k=1}^{\infty} a_{k} r^{k} \geq \frac{\left|a_{-p}\right|}{r^{p}}-r \sum_{k=1}^{\infty} a_{k} \\
& \geq \frac{\left|a_{-p}\right|}{r^{p}}-\frac{(B-A)(p-\alpha)\left|a_{-p}\right|}{\left[\frac{\ell}{\ell+\lambda(1+p)}\right]^{m+1}[(1+p)(1+B)+(A-B)(p-\alpha)]} r,
\end{aligned}
$$

which gives the left hand side of (3.1). It can be easily seen that the function $f(z)$ defined by (3.2) is an extremal function for the theorem. 
Theorem 4. If $f(z)=\frac{a_{-p}}{z^{p}}+\sum_{k=1}^{\infty}(-1)^{k-1} a_{k} z^{k}\left(a_{-p} \neq 0 ; a_{k} \geq 0, p \in \mathbb{N}\right)$ is in the class $\sum_{a}^{*}(p, m, \alpha, \lambda, \ell, A, B)$, then for $0<|z|=r<1$,

$$
\begin{aligned}
& \frac{p\left|a_{-p}\right|}{r^{p+1}}-\frac{(B-A)(p-\alpha)\left|a_{-p}\right|}{\left[\frac{\ell}{\ell+\lambda(1+p)}\right]^{m+1}[(1+p)(1+B)+(A-B)(p-\alpha)]} \leq\left|f^{\prime}(z)\right| \\
& \leq \frac{p\left|a_{-p}\right|}{r^{p+1}}+\frac{(B-A)(p-\alpha)\left|a_{-p}\right|}{\left[\frac{\ell}{\ell+\lambda(1+p)}\right]^{m+1}[(1+p)(1+B)+(A-B)(p-\alpha)]} .
\end{aligned}
$$

The result is sharp, the extremal function being of the form (3.2).

Proof. From Theorem 2, we have

$$
\begin{aligned}
& {\left[\frac{\ell}{\ell+\lambda(1+p)}\right]^{m+1}[(1+p)(1+B)+(A-B)(p-\alpha)] \sum_{k=1}^{\infty} k a_{k}} \\
& \quad \leq \sum_{k=1}^{\infty}\left[\frac{\ell}{\ell+\lambda(1+p)}\right]^{m+1}[(1+p)(1+B)+(A-B)(p-\alpha)] a_{k} \\
& \quad \leq(B-A)(p-\alpha)\left|a_{-p}\right|,
\end{aligned}
$$

which evidently yields

$$
\sum_{k=1}^{\infty} k a_{k} \leq \frac{(B-A)(p-\alpha)\left|a_{-p}\right|}{\left[\frac{\ell}{\ell+\lambda(1+p)}\right]^{m+1}[(1+p)(1+B)+(A-B)(p-\alpha)]} .
$$

Consequently, we obtain

$$
\begin{aligned}
\left|f^{\prime}(z)\right| & \leq \frac{p\left|a_{-p}\right|}{r^{p+1}}+\sum_{k=1}^{\infty} k a_{k} r^{k-1} \leq \frac{p\left|a_{-p}\right|}{r^{p+1}}+\sum_{k=1}^{\infty} k a_{k} \\
& \leq \frac{p\left|a_{-p}\right|}{r^{p+1}}+\frac{(B-A)(p-\alpha)\left|a_{-p}\right|}{\left[\frac{\ell}{\ell+\lambda(1+p)}\right]^{m+1}[(1+p)(1+B)+(A-B)(p-\alpha)]}
\end{aligned}
$$

by (3.5). Also

$$
\begin{aligned}
& \left|f^{\prime}(z)\right| \geq \frac{p\left|a_{-p}\right|}{r^{p+1}}-\sum_{k=1}^{\infty} k a_{k} r^{k-1} \geq \frac{p\left|a_{-p}\right|}{r^{p+1}}-\sum_{k=1}^{\infty} k a_{k} \\
& \geq \frac{p\left|a_{-p}\right|}{r^{p+1}}-\frac{(B-A)(p-\alpha)\left|a_{-p}\right|}{\left[\frac{\ell}{\ell+\lambda(1+p)}\right]^{m+1}[(1+p)(1+B)+(A-B)(p-\alpha)]} .
\end{aligned}
$$


This completes the proof of Theorem 4 .

\section{Closure theorems}

Let the functions $f_{j}(z)$ be defined for $j=1,2, \ldots, m$, by

$$
f_{j}(z)=\frac{a_{-p, j}}{z^{p}}+\sum_{k=1}^{\infty}(-1)^{k-1} a_{k, j} z^{k}\left(a_{-p, j}>0 ; a_{k, j} \geq 0 ; p \in \mathbb{N}\right) \text { for } z \in U^{*} .
$$

Theorem 5. Let the function $f_{j}(z)$ be defined by (4.1) be in the class $\sum_{a}^{*}(p, m, \alpha, \lambda, \ell, A, B)$ for every $j=1,2, \ldots, m$. Then the function $F(z)$ defined by

$$
F(z)=\frac{b_{-p}}{z^{p}}+\sum_{k=1}^{\infty}(-1)^{k-1} b_{k} z^{k} \quad\left(b_{-p}>0 ; b_{k} \geq 0 ; p \in \mathbb{N}\right),
$$

is a member of the class $\sum_{a}^{*}(p, m, \alpha, \lambda, \ell, A, B)$, where

$$
b_{-p}=\frac{1}{m} \sum_{j=1}^{m} a_{-p, j} \quad \text { and } \quad b_{k}=\frac{1}{m} \sum_{j=1}^{m} a_{k, j}(k \in N) .
$$

Proof. Since $f_{j}(z) \in \sum_{a}^{*}(p, m, \alpha, \lambda, \ell, A, B)$, it follows from Theorem 2 , that

$$
\begin{aligned}
& \sum_{k=1}^{\infty}\left[\frac{\ell}{\ell+\lambda(k+p)}\right]^{m+1}[(k+p)(1+B)+(A-B)(p-\alpha)] a_{k, j} \\
& \quad \leq(B-A)(p-\alpha)\left|a_{-p, j}\right|,
\end{aligned}
$$

for every $j=1,2, \ldots, m$. Hence,

$$
\begin{aligned}
& \sum_{k=1}^{\infty}\left[\frac{\ell}{\ell+\lambda(k+p)}\right]^{m+1}[(k+p)(1+B)+(A-B)(p-\alpha)] b_{k} \\
& \quad=\sum_{k=1}^{\infty}\left[\frac{\ell}{\ell+\lambda(k+p)}\right]^{m+1}[(k+p)(1+B)+(A-B)(p-\alpha)]\left\{\frac{1}{m} \sum_{j=1}^{\infty} a_{k, j}\right\} \\
& \quad=\frac{1}{m} \sum_{j=1}^{m}\left(\sum_{k=1}^{\infty}\left[\frac{\ell}{\ell+\lambda(k+p)}\right]^{m+1}[(k+p)(1+B)+(A-B)(p-\alpha)] a_{k, j}\right) \\
& \quad \leq(B-A)(p-\alpha)\left(\frac{1}{m} \sum_{j=1}^{\infty} a_{-p, j}\right)=(B-A)(p-\alpha) b_{-p},
\end{aligned}
$$

which (in view of Theorem 2) implies that $F(z) \in \sum_{a}^{*}(p, m, \alpha, \lambda, \ell, A, B)$.

Theorem 6. The class $\sum_{a}^{*}(p, m, \alpha, \lambda, \ell, A, B)$ is closed under convex linear combination. 
Proof. Let the functions $f_{j}(z)(j=1,2)$ defined by (4.1) be in the class $\sum_{a}^{*}(p, m, \alpha, \lambda, \ell, A, B)$, it is sufficient to prove that the function

$$
H(z)=t f_{1}(z)+(1-t) f_{2}(z) \quad(0 \leq t \leq 1)
$$

is also in the class $\sum_{a}^{*}(p, m, \alpha, \lambda, \ell, A, B)$. Since for $0 \leq t \leq 1$,

$$
H(z)=\frac{t a_{-p, 1}+(1-t) a_{-p, 2}}{z^{p}}+\sum_{k=1}^{\infty}(-1)^{k-1}\left\{t a_{k, 1}+(1-t) a_{k, 2}\right\} z^{k},
$$

we observe that

$$
\begin{aligned}
& \sum_{k=1}^{\infty}\left[\frac{\ell}{\ell+\lambda(k+p)}\right]^{m+1}[(k+p)(1+B)+(A-B)(p-\alpha)]\left\{t a_{k, 1}+(1-t) a_{k, 2}\right\} \\
& =t \sum_{k=1}^{\infty}\left[\frac{\ell}{\ell+\lambda(k+p)}\right]^{m+1}[(k+p)(1+B)+(A-B)(p-\alpha)] a_{k, 1}+ \\
& (1-t) \sum_{k=1}^{\infty}\left[\frac{\ell}{\ell+\lambda(k+p)}\right]^{m+1}[(k+p)(1+B)+(A-B)(p-\alpha)] a_{k, 2} \\
& \leq(B-A)(p-\alpha)\left\{t a_{-p, 1}+(1-t) a_{-p, 2}\right\},
\end{aligned}
$$

with the aid of Theorem 2, hence $H(z) \in \sum_{a}^{*}(p, m, \alpha, \lambda, \ell, A, B)$. This completes the proof of Theorem 6.

Theorem 7. Let

$$
f_{0}(z)=\frac{a_{-p}}{z^{p}}
$$

and

$$
f_{k}(z)=\frac{a_{-p}}{z^{p}}+(-1)^{k-1} \frac{(B-A)(p-\alpha)\left|a_{-p}\right|}{\left[\frac{\ell}{\ell+\lambda(k+p)}\right]^{m+1}\{(k+p)(1+B)+(A-B)(p-\alpha)\}} z^{k} \quad(k \in N) .
$$

Then $f(z) \in \sum_{a}^{*}(p, m, \alpha, \lambda, \ell, A, B)$ if and only if it can be expressed in the form

$$
f(z)=\sum_{k=0}^{\infty} \mu_{k} f_{k}(z), \text { where } \mu_{k} \geq 0 \quad(k \geq 0) \text { and } \sum_{k=0}^{\infty} \mu_{k}=1
$$

Proof. Suppose that

$$
f(z)=\sum_{k=0}^{\infty} \mu_{k} f_{k}(z) \text {, where } \mu_{k} \geq 0 \quad(k \geq 0) \text { and } \sum_{k=0}^{\infty} \mu_{k}=1 .
$$

Then 


$$
\begin{aligned}
f(z) & =\sum_{k=0}^{\infty} \mu_{k} f_{k}(z) \\
& =\mu_{0} f_{0}(z)+\sum_{k=1}^{\infty} \mu_{k} f_{k}(z) \\
& =\frac{a_{-p}}{z^{p}}+\sum_{k=1}^{\infty} \mu_{k}(-1)^{k-1} \frac{(B-A)(p-\alpha)\left|a_{-p}\right|}{\left[\frac{\ell}{\ell+\lambda(k+p)}\right]^{m+1}[(k+p)(1+B)+(A-B)(p-\alpha)]} z^{k}(k \in N)
\end{aligned}
$$

Since

$$
\begin{aligned}
& \sum_{k=1}^{\infty}\left[\frac{\ell}{\ell+\lambda(k+p)}\right]^{m+1}\{(k+p)(1+B)+(A-B)(p-\alpha)\} \\
& {\left[\frac{\ell}{\ell+\lambda(k+p)}\right]^{m+1}\{(k+p)(1+B)+(A-B)(p-\alpha)\}} \\
& \quad=(B-A)(p-\alpha)\left|a_{-p}\right| \sum_{k=1}^{\infty} \mu_{k} \\
& \quad=(B-A)(p-\alpha)\left|a_{-p}\right|\left(1-\mu_{0}\right) \\
& \quad \leq(B-A)(p-\alpha)\left|a_{-p}\right|
\end{aligned}
$$

we have $f(z) \in \sum_{a}^{*}(p, m, \alpha, \lambda, \ell, A, B)$, by Theorem 2 .

Conversely, suppose that the function $f(z)$ defined by (1.9) belongs to the class $\sum_{a}^{*}(p, m, \alpha, \lambda, \ell, A, B)$. Since

$$
a_{k} \leq \frac{(B-A)(p-\alpha)\left|a_{-p}\right|}{\left[\frac{\ell}{\ell+\lambda(k+p)}\right]^{m+1}\{(k+p)(1+B)+(A-B)(p-\alpha)\}} \quad(k \in N),
$$

by Corollary 1 , setting

$$
\mu_{k}=\frac{\left[\frac{\ell}{\ell+\lambda(k+p)}\right]^{m+1}\{(k+p)(1+B)+(A-B)(p-\alpha)\}}{1 / \ell(A-B)(p-\alpha)\left|a_{-p}\right|} a_{k} \quad(k \in N),
$$

and

$$
\mu_{0}=1-\sum_{k=1}^{\infty} \mu_{k} f_{k}(z)
$$

it follows that

$$
f(z)=\sum_{k=0}^{\infty} \mu_{k} f_{k}(z)
$$

This completes the proof of Theorem 7. 


\section{Integral operators}

Theorem 8. If $f(z)=\frac{a_{-p}}{z^{p}}+\sum_{k=1}^{\infty}(-1)^{k-1} a_{k} z^{k}\left(a_{-p} \neq 0 ; a_{k} \geq 0, p \in \mathbb{N}\right)$ is in the class $\sum_{a}^{*}(p, m, \alpha, \lambda, \ell, A, B)$, then

$$
\begin{aligned}
F(z) & =(c-p+1) z^{-c-1} \int_{0}^{z} t^{c} f(t) d t \\
& =\frac{a_{-p}}{z^{p}}+\sum_{k=1}^{\infty}(-1)^{k-1}\left(\frac{c-p+1}{c+k+1}\right) a_{k} z^{k},
\end{aligned}
$$

$c>p-1$, belongs to the class $\sum_{a}^{*}(p, m, \gamma(p, \alpha, c, A, B), \lambda, \ell, A, B)$, where

$$
\gamma(p, \alpha, c, A, B)=p-\frac{(c-p+1)(1+p)(1+B)(p-\alpha)}{(c+p+1)[(1+p)(1+B)+(A-B)(p-\alpha)]-(c-p+1)(A-B)(p-\alpha)} .
$$

The result is sharp for the function $f(z)$ given by

$$
f(z)=\frac{a_{-p}}{z^{p}}+\frac{(B-A)(p-\alpha)\left|a_{-p}\right|}{\left[\frac{\ell}{\ell+\lambda(1+p)}\right]^{m+1}[(1+p)(1+B)+(A-B)(p-\alpha)]} z .
$$

Proof. Suppose $f(z)=\frac{a_{-p}}{z^{p}}+\sum_{k=1}^{\infty}(-1)^{k-1} a_{k} z^{k} \in \sum_{a}^{*}(p, m, \alpha, \lambda, \ell, A, B)$, in view of Theorem 2, we shall find the largest value of $\gamma$ for which

$$
\sum_{k=1}^{\infty} \frac{\left[\frac{\ell}{\ell+\lambda(k+p)}\right]^{m+1}[(k+p)(1+B)+(A-B)(p-\gamma)]}{(B-A)(p-\alpha)\left|a_{-p}\right|}\left(\frac{c-p+1}{c+k+1}\right) a_{k} \leq 1 .
$$

It is sufficient to fined the range values of $\gamma$ for which

$$
\frac{(c-p+1)[(k+p)(1+B)+(A-B)(p-\gamma)]}{(c+k+1)(p-\gamma)} \leq \frac{[(k+p)(1+B)+(A-B)(p-\alpha)]}{(p-\alpha)}
$$

for each $k$.

Solving the above inequality for $\gamma$, we obtain

$$
\gamma \leq p-\frac{(c-p+1)(k+p)(1+B)(p-\alpha)}{(c+k+1)[(k+p)(1+B)+(A-B)(p-\alpha)]-(c-p+1)(A-B)(p-\alpha)} .
$$

For each $p, \alpha, \lambda, \ell, A, B$ and $c$ fixed let

$$
F(k)=p-\frac{(c-p+1)(k+p)(1+B)(p-\alpha)}{(c+k+1)[(k+p)(1+B)+(A-B)(p-\alpha)]-(c-p+1)(A-B)(p-\alpha)} .
$$

Then $F(k+1)-F(k)=\frac{D}{G}>0$ for each $k$, where

$$
D=(c-p+1)\left(1+B^{2}\right)(p-\alpha)(k+p)(k+p+1)
$$


and

$$
\begin{aligned}
G= & \{(c+k+2)[(k+p+1)(1+B)+(A-B)(p-\alpha)]-(c-p+1)(A-B)(p-\alpha)\} \\
& \times\{(c+k+1)[(k+p)(1+B)+(A-B)(p-\alpha)]-(c-p+1)(A-B)(p-\alpha)\} .
\end{aligned}
$$

Hence $F(k)$ is an increasing function of $k$. Since

$$
F(1)=p-\frac{(c-p+1)(1+p)(1+B)(p-\alpha)}{(c+2)[(1+p)(1+B)+(A-B)(p-\alpha)]-(c-p+1)(A-B)(p-\alpha)},
$$

the result follows.

\section{Remark.}

(i) Putting $a_{-p}=1, p=1, m=0, A=-1$ and $B=1$, in the above results, we have the results obtained by Uralegaddi and Ganigi [11];

(ii) Putting $a_{-p}=1, p=1, A=-1$ and $B=1$, in the above results, we have the results obtained by Aouf and Darwish [1];

(iii) Putting $a_{-p}=1$ and $p=1$, in the above results, we have the reults obtained by Aouf et al. $[2]$.

\section{Convolution properties}

Theorem 9. If $f(z)$ and $g(z)$ belong to the class $B_{m}(p, \alpha, \lambda, \ell, A, B)$, then

$$
T(z)=\frac{a_{-p} b_{-p}}{z^{p}}+\sum_{k=1}^{\infty}\left(a_{k}^{2}+b_{k}^{2}\right) z^{k}
$$

is in the class $B_{m}\left(p, \alpha, \lambda, \ell, A_{1}, B_{1}\right)$ such that $A_{1}<-\mu^{2}+B_{1}\left(1-\mu^{2}\right)$, where

$$
\mu=\frac{\sqrt{2(p-\alpha)(k+p)\left(\left|a_{-p}\right|\left|b_{-p}\right|\right)(B-A)}}{\sqrt{2\left|a_{-p}\right|\left|b_{-p}\right|}(B-A)(p-\alpha)+\sqrt{\left[\frac{\ell}{\ell+\lambda(k+p)}\right]^{m+1}[(k+p)(1+B)+(A-B)(p-\alpha)]}} .
$$

Proof. Since $f, g \in B_{m}(p, \alpha, \lambda, \ell, A, B)$. Theorem 2 yields

$$
\sum_{k=1}^{\infty}\left(\frac{\left[\frac{\ell}{\ell+\lambda(k+p)}\right]^{m+1}[(k+p)(1+B)+(A-B)(p-\alpha)] a_{k}}{(B-A)(p-\alpha)\left|a_{-p}\right|}\right)^{2} \leq 1,
$$

and

$$
\sum_{k=1}^{\infty}\left(\frac{\left[\frac{\ell}{\ell+\lambda(k+p)}\right]^{m+1}[(k+p)(1+B)+(A-B)(p-\alpha)] b_{k}}{(B-A)(p-\alpha)\left|b_{-p}\right|}\right)^{2} \leq 1
$$


we obtain from the last two inequalities

$$
\sum_{k=1}^{\infty} \frac{1}{2}\left(\frac{\left[\frac{\ell}{\ell+\lambda(k+p)}\right]^{m+1}[(k+p)(1+B)+(A-B)(p-\alpha)]}{(B-A)(p-\alpha)\left|a_{-p}\right|\left|b_{-p}\right|}\right)^{2}\left(a_{k}^{2}+b_{k}^{2}\right) \leq 1,
$$

However, $T(z) \in B_{m}\left(p, \alpha, \lambda, \ell, A_{1}, B_{1}\right)$ if and only if

$$
\sum_{k=1}^{\infty}\left(\frac{\left[\frac{\ell}{\ell+\lambda(k+p)}\right]^{m+1}\left[(k+p)\left(1+B_{1}\right)+\left(A_{1}-B_{1}\right)(p-\alpha)\right]}{\left(B_{1}-A_{1}\right)(p-\alpha)\left|a_{-p}\right|\left|b_{-p}\right|}\right)\left(a_{k}^{2}+b_{k}^{2}\right) \leq 1 .
$$

where $-1 \leq A_{1}<B_{1} \leq 1, \lambda \geq 0, \ell>0$, but (6.2) implies (6.3) if

$$
\begin{gathered}
\frac{\left[\frac{\ell}{\ell+\lambda(k+p)}\right]^{m+1}\left[(k+p)\left(1+B_{1}\right)+\left(A_{1}-B_{1}\right)(p-\alpha)\right]}{\left(B_{1}-A_{1}\right)(p-\alpha)\left|a_{-p}\right|\left|b_{-p}\right|} \\
\quad<\frac{1}{2}\left(\frac{\left[\frac{\ell}{\ell+\lambda(k+p)}\right]^{m+1}[(k+p)(1+B)+(A-B)(p-\alpha)]}{(B-A)(p-\alpha)\left|a_{-p}\right|\left|b_{-p}\right|}\right)^{2} .
\end{gathered}
$$

Hence, if

$$
\frac{1+B_{1}}{B_{1}-A_{1}}<\frac{2(B-A)^{2}(p-\alpha)^{2}\left|a_{-p}\right|\left|b_{-p}\right|+\left[\frac{\ell}{\ell+\lambda(k+p)}\right]^{m+1}[(k+p)(1+B)+(A-B)(p-\alpha)]^{2}}{2(B-A)^{2}(p-\alpha)(k+p)\left|a_{-p}\right|\left|b_{-p}\right|} .
$$

This is equivalent to

$$
\frac{B_{1}-A_{1}}{1+B_{1}}>\frac{2(B-A)^{2}(p-\alpha)(k+p)\left|a_{-p}\right|\left|b_{-p}\right|}{2(B-A)^{2}(p-\alpha)^{2}\left|a_{-p}\right|\left|b_{-p}\right|+\left[\frac{\ell}{\ell+\lambda(k+p)}\right]^{m+1}[(k+p)(1+B)+(A-B)(p-\alpha)]^{2}}=\mu^{2} .
$$

Hence we get $A_{1}<-\mu^{2}+B_{1}\left(1-\mu^{2}\right)$.

Theorem 10. Let $f(z)$ and $g(z)$ belong to the class $B_{m}(p, \alpha, \lambda, \ell, A, B)$. Then the convolution (or Hadamard product) of two functions $f$ and $g$ belong to the class that $i s,(f * g)(z) \in B_{m}(p, \alpha, \lambda, \ell$, $\left.A_{1}, B_{1}\right)$, where $A_{1}<-v+B_{1}(1-v)$ and

$$
v=\frac{(B-A)^{2}(p-\alpha)\left|a_{-p}\right|\left|b_{-p}\right|}{(B-A)^{2}(p-\alpha)^{2}\left|a_{-p}\right|\left|b_{-p}\right|+\left[\frac{\ell}{\ell+\lambda(k+p)}\right]^{m+1}[(k+p)(1+B)+(A-B)(p-\alpha)]^{2}} .
$$


Proof. Since $f, g \in B_{m}(p, \alpha, \lambda, \ell, A, B)$, by using the Cauchy-Schwarz inequality and Theorem 2 , we obtain

$$
\begin{aligned}
\sum_{k=1}^{\infty} & \frac{\left[\frac{\ell}{\ell+\lambda(k+p)}\right]^{m+1}[(k+p)(1+B)+(A-B)(p-\alpha)]}{(B-A)(p-\alpha)\left|a_{-p}\right|\left|b_{-p}\right|} \\
& \leq\left(\sum_{k=1}^{\infty} \frac{\left[\frac{\ell}{\ell+\lambda(k+p)}\right]^{m+1}[(k+p)(1+B)+(A-B)(p-\alpha)]}{(B-A)(p-\alpha)\left|a_{-p}\right|} a_{k}\right)^{1 / 2} \\
& \cdot\left(\sum_{k=1}^{\infty} \frac{\left[\frac{\ell}{\ell+\lambda(k+p)}\right]^{m+1}[(k+p)(1+B)+(A-B)(p-\alpha)]}{(B-A)(p-\alpha)\left|b_{-p}\right|} b_{k}\right)^{1 / 2} \\
& \leq 1,
\end{aligned}
$$

we must fined the values of $A_{1}, B_{1}$ so that

$$
\sum_{k=1}^{\infty} \frac{\left[\frac{\ell}{\ell+\lambda(k+p)}\right]^{m+1}\left[(k+p)\left(1+B_{1}\right)+\left(A_{1}-B_{1}\right)(p-\alpha)\right]}{\left(B_{1}-A_{1}\right)(p-\alpha)\left|a_{-p}\right|\left|b_{-p}\right|} a_{k} b_{k}<1 .
$$

Therefor, by (6.5), (6.6) holds true if

$$
\sqrt{a_{k} b_{k}} \leq \frac{\left(B_{1}-A_{1}\right)[(k+p)(1+B)+(A-B)(p-\alpha)]}{(B-A)\left[(k+p)\left(1+B_{1}\right)+\left(A_{1}-B_{1}\right)(p-\alpha)\right]},
$$

$k \geq m, m \geq p, a_{k} \neq 0, b_{k} \neq 0$.

By (6.5), we have

$$
\sqrt{a_{k} b_{k}}<\frac{(B-A)(p-\alpha)\left|a_{-p}\right|}{\left[\frac{\ell}{\ell+\lambda(k+p)}\right]^{m+1}[(k+p)(1+B)+(A-B)(p-\alpha)]},
$$

therefor (6.7) holds true

if

$$
\begin{gathered}
\frac{\left[\frac{\ell}{\ell+\lambda(k+p)}\right]^{m+1}\left[(k+p)\left(1+B_{1}\right)+\left(A_{1}-B_{1}\right)(p-\alpha)\right]}{\left(B_{1}-A_{1}\right)(p-\alpha)\left|a_{-p}\right|\left|b_{-p}\right|} \\
\quad \leq\left[\frac{\left[\frac{\ell}{\ell+\lambda(k+p)}\right]^{m+1}[(k+p)(1+B)+(A-B)(p-\alpha)]}{(B-A)(p-\alpha)\left|a_{-p}\right|\left|b_{-p}\right|}\right]^{2},
\end{gathered}
$$


which is equivalent to

$$
\frac{1+B_{1}}{B_{1}-A_{1}}<\frac{(B-A)^{2}(p-\alpha)^{2}\left|a_{-p}\right|\left|b_{-p}\right|+\left[\frac{\ell}{\ell+\lambda(k+p)}\right]^{m+1}[(k+p)(1+B)+(A-B)(p-\alpha)]^{2}}{(B-A)^{2}(p-\alpha)\left|a_{-p}\right|\left|b_{-p}\right|} .
$$

Alternatively, we can write

$$
\frac{B_{1}-A_{1}}{1+B_{1}}>\frac{(B-A)^{2}(p-\alpha)\left|a_{-p}\right|\left|b_{-p}\right|}{(B-A)^{2}(p-\alpha)^{2}\left|a_{-p}\right|\left|b_{-p}\right|+\left[\frac{\ell}{\ell+\lambda(k+p)}\right]^{m+1}[(k+p)(1+B)+(A-B)(p-\alpha)]^{2}}=v .
$$

Hence we get $A_{1}<-v+B_{1}(1-v)$.

\section{References}

[1] M. K. Aouf and H. E. Darwish, Meromorphic starlike univalent functions with alternating coefficients, Utilitas Math., 47 (1995), 137-144.

[2] M. K. Aouf, H. M. Hossen and A. Y. Lashin, A certain subclass of meromorphically starlike functions with alternating coefficients, Math. Cluj, 44 (67) (2002), 3-10.

[3] E. Aqlan, J. M. Jahangiri and S. R. Kulkarni, Certain integral operators applied to meromorphic p-valent functions, J. Nat. Geom., 24(2003), 111-120.

[4] R. M. El-Ashwah, Properties of certain class of p-valent meromorphic functions associated with new integral operator, Acta Universitatis Apulensis, 29 (2012), 255-264.

[5] R. M. El-Ashwah and M. K. Aouf, Applications of differential subordination on certain class of meromorphic p-valent functions associated with certain integral operator, Acta Universitatis Apulensis, 31 (2012), 53-63.

[6] A. Y. Lashin, On certain subclass of meromorphic functions associated with certain integral operators, Comput. Math. Appl., 59 (1) (2010), 524-531.

[7] S. S. Miller and P. T. Mocanu, Differential subordination and univalents functions, Michigan Math. J., 28 (1981), 157-171.

[8] S. S. Miller and P. T. Mocanu, Differential subordination: Theory and Applications, Series on Monographs and Textbooks in Pure and Applied Mathematics, Vol. 225. Marcel Dekker, New York and Basel, 2000.

[9] Sh. Najafzadeh, A. Ebadian and S. Shams, P-valent meromorphic functions with alternating coefficients based on integral operator, J. Inequal. pure and Appl. Math., 9 (2) (2008), Art. 40, 5pp.

[10] H. Silverman, Univalent functions with negative coefficients, Proc. Amer. Math. Soc., 51(1975), $109-116$.

[11] B. A. Uralegaddi and M. D. Ganigi, Meromorphic starlike functions with alternating coefficients, Rend. Mat., 11 (7) (1991), 441-446.

Department of Mathematics, Faculty of Science, Mansoura, University, Mansoura, Egypt.

E-mail: Darwish333@yahoo.com

E-mail: aylashin@yahoo.com

E-mail: bakeralhaaiti@yahoo.com 